\title{
THE INTRAFAMILIAL TRANSMISSION OF RHEUMATOID ARTHRITIS-V
}

\section{DIFFERENCES BETWEEN RHEUMATOID ARTHRITICS AND CONTROLS ON SELECTED PERSONALITY VARIABLES}

\author{
Stanislav V. Kasl, Ph.D. and Sidney CobB, M.D., M.P.H. \\ Institule for Sucial Research and the Department of Psychology, The University of Michigan, Ann \\ Arbor, Michigan
}

(Received 21 December 1967; in revised form 26 August 1968)

IT Is the purpose of this report to present findings which describe some differences in the adult personalities of rheumatoid arthritics and controls. The broad hypotheses investigated in this part of the study derive from an examination of the relevant research literature and, in particular, the several excellent reviews available [1-3]. Specifically, the intent is to provide an adequate description, based on the subjects' own reports, of three general areas of psychological functioning: (1) symptoms broadly indicative of poor mental health; (2) diverse affective responses; and (3) expression and control of aggression and hostility.

Clearly, this part of the study does not seek to confirm a single, focal hypothesis. Rather, its intent is broadly descriptive: it seeks to contribute to the accumulating, but sometimes confusing and inconsistent evidence regarding possible differences between arthritics and controls. Since one of the more confusing areas is the one dealing with expression and control of aggression and hostility, this study accordingly develops a number of different measures in order to achieve a multiple, convergent operationalism (methodological triangulation) as advocated by CAMPBELL and FiSKE [4]. In addition, special emphasis will be given to patterns of sex differences, another frequent source of confusion [5]. Finally, we shall pay attention to any possible differences between national and clinic samples, inasmuch as source of subjects is another probable reason for inconsistencies in results [2].

\section{METHODS}

Since the relevant details of the design of the study and of some of the measures have been presented in the first 2 reports in this series, this section will describe only the measurement procedures for the adult personality variables.

Four indices were constructed from a checklist of 20 symptoms, used previously in a national interview study of mental health [6]. The index construction was based on the factor analysis done by the original authors and closely parallels their own indices; the labelling of the indices is theirs. 
(1) Physical ill health-5 items dealing with the frequency of occurrence of minor symptoms, such as cough, sore throat, running nose, or pain in the chest.

(2) Physical anxiety-3 items asking about the frequency of physical symptoms presumed to reflect anxiety: shortness of breath, diarrhea, and heart beating hard.

(3) Psychological anxiety -10 items asking about feelings tense, nervous, and anxious, plus other questions on appetite, digestion, and sleeping.

(4) Immobilization-3 items reflecting feelings of being tired out and fatigued.

A number of additional indices were constructed, all pertinent to a broad conception of mental health [7-9].

(5) Depression-8 items asking about feelings of sadness, unhappiness, unworthiness, dissatisfaction with life, etc.

(6) Self-esteem (adult) -8 items in which respondents evaluate themselves and their performance in a number of typical social roles, such as spouse, parent, provider or housekeeper, etc.; items are different for males and for females.

(7) Self-confidence - 14 items raising a number of questions about stability of selfesteem, vulnerability to criticism, tendency to blame oneself, etc.

(8) Job satisfaction -6 items; for males, they were broad questions on satisfaction with job, with promotion, job performance, etc. For females, the items deal primarily with satisfaction with the role of housewife.

(9) Desire for change-5 items asking respondents if they would like to change themselves in different areas, such as skills, habits, family relations, etc. The scale was previously used by CoBB et al. [10].

(10) Impulsiveness-5 items dealing with such behaviors as acting on impulse and inability to control sudden temptations.

Another set of scales dealt with the area of anger-aggression and its control.

(11) Frequency of anger-irritation (general)- 7 items about irritability, frequency of being annoyed, etc.

(12) Frequency of anger-irritation (spouse-specific)-4 items, similar to those above, except that the respondent's spouse is specified as the source or the target.

(13) Impulse to overt aggression (general) - 5 items asking the respondent how often he feels like being rude to someone, picking a fight, smashing things, etc.

(14) Frequency of overt aggression (general) -5 items asking how often he actually does engage in the above behaviors.

(15) and (16) Impulse to overt aggression (spouse-specific) and frequency of overt aggression (spouse-specific) - 2 items in each scale; two of the same items as in the the above 2 scales, except that the respondent's spouse is the specified target of the impulse or the actual aggressive behavior.

(17) Duration of anger-4 items inquiring about how long it takes for anger or angry thoughts to dissipate.

(18) Guilt about spouse-directed aggression-5 items about values against expressing aggression towards spouse and about amount of guilt felt.

(19) Interviewer's ratings of respondent's irritation-3 items; at the end of each interview, the interviewer rated the subject's amount of irritation.

Finally, two measures were constructed to assess motivational predispositions in the area of independence-dependence. The scales are based on a factor analysis of a large pool of items [11] and on previous work done with the items [12]. 
(20) Need for independence (general)-5 items which reflect efforts to be free of the possible or actual control of others.

(21) Need for emotional dependence (general) - 5 items indicating the need to receive emotional support and encouragement from others.

\section{RESULTS}

In this section, we shall consider the following substantive areas: (1) Diverse mental health correlates of RA; (2) The association of RA with several indicators of strength of aggressive impulses, frequency of angry and aggressive behavior, and irritability; (3) The association of RA with derived indices of conflict over expression of aggression; (4) Correlates of RA with the husband-wife pairs as units of analysis.

In much of the data presentation which follows, we shall be examining differences between RA's and non-RA's and this will be done separately for men and for women. Moreover, we shall be on the lookout for possible effects of source of sample, National vs. Clinic, and for a sex-disease interaction (i.e., is the difference between RA's and non-RA's the same for men as it is for women). For purposes of overview, Table 1 gives the case frequencies according to sex, disease, and source of sample. To be sure, in a particular analysis, the frequencies may be slightly smaller because of missing data on a few subjects.

Table 1. Distribution of cases according to seX, Disease and source of the sample

\begin{tabular}{lcccccc}
\hline & \multicolumn{2}{c}{ Women } & & \multicolumn{2}{c}{ Men } \\
\cline { 2 - 3 } \cline { 5 - 6 } & $\begin{array}{c}\text { RA } \\
\text { present }\end{array}$ & $\begin{array}{c}\text { RA } \\
\text { absent }\end{array}$ & & $\begin{array}{c}\text { RA } \\
\text { present }\end{array}$ & $\begin{array}{c}\text { RA } \\
\text { absent }\end{array}$ \\
\hline National sample & 28 & 59 & & 5 & 92 \\
Clinic sample & 21 & 47 & & 12 & 60 \\
\hline Total & 49 & 106 & & 17 & 152 \\
\hline
\end{tabular}

Table 2 presents the data on the association of RA with a number of mental health variables. First, the mean scores are presented so that the reader can see in which direction the differences lie. The scales are labelled in such a way that the higher the score on a scale, the more of the implied behavior is reported by the subjects. Because the scales vary in means and standard deviations, and because tests of significance do not indicate the strength of the association, the values for 2 measures of association are presented, gamma and Tau. The rationale for choosing these measures was discussed in the fourth report in this series, dealing with the findings on the perception of parents. The table also gives information on differences between National and Clinic samples; any differences between means significant at the 0.05 level, labelled 'Yes' in the table, will be noted and adjustments carried out and discussed. Finally, the last column of the table gives data on the significance of sex-discasc intcraction. Basically, it involves testing for the significance of a difference between differences in two pairs of means; for example, for the first row variable it would read: (3.96$3.40)-(4.71-3.72)$. The procedure uses a single estimate of population variance, derived from the 'within variance' term in a one-way analysis of variance; for greater details, see SCOTT and WeRTHEIMER [13]. 


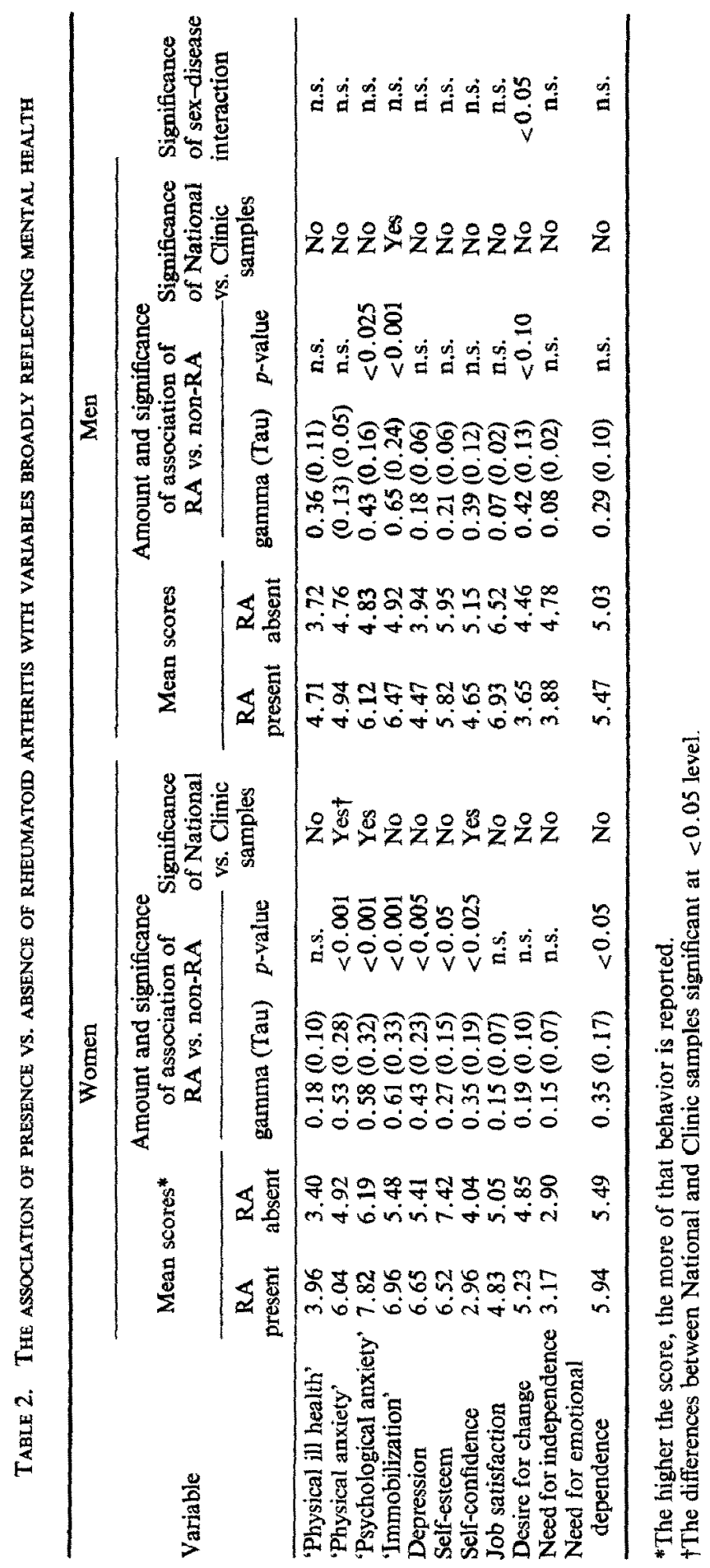


The findings in Table 2 may be summarized as follows. Among the women, the RA's are significantly higher on physical and psychological anxiety and immobilization; moreover, they describe themselves as more depressed, more dissatisfied with themselves, less self-confident, and somewhat more emotionally dependent on others. Among the men, the RA's are significantly higher on only two scales, psychological anxiety and immobilization. However, most of the other differences between male RA's and non-RA's are in the same direction as for the women, and only one sexdisease interaction is significant: male arthritics report less of a desire to see a change in themselves than healthy males, whereas female arthritics report more of a desire for change than healthy females. The desire for change measure was used in a previous study [10], where it was found that among male patients (including arthritics) there was a mild positive association between self-evaluation and desire for change, whereas among controls there was found the more expected negative association between the same two variables. Similarly, in the present study, healthy males who are lower on self-esteem tend to report a greater desire for change $(r=-0.39$, $p<0.001$ ), whereas among the arthritics there is a nonsignificant correlation in the opposite direction $(r=0.28)$; the difference in the correlations is significant $(p<0.01)$.

It can be seen that in Table 2 there are 4 scales for which there was a significant sample difference. The procedure adopted to adjust for these differences was to convert, within each sample, raw scores into standard scores and then recompute the gamma and Tau statistics on these standard scores. The new gamma and Tau values, revealed that these corrections make little practical difference, and in 3 out of 4 cases result in slightly higher values, i.e. bigger differences between RA's and non-RA's.

We shall next turn our attention to a number of indicators of strength of aggressive impulses, frequency of angry and aggressive behavior, and irritability. The basic findings are given in Table 3 and may be summarized as follows. Women with RA report a greater frequency of anger-irritation (non-specific anger as well as anger directed at their husbands) and stronger impulses to overt aggression (general and husband-directed), but are higher on actual amount of overt aggression only when it is directed at their spouse. In addition, the interviewers rated women with RA as somewhat more irritable. With respect to duration of anger and guilt about husbanddirected aggression women with RA were not significantly different from women without RA. Men with RA reported a weaker impulse to overt aggression (general), less frequent aggression dircetcd at their wives and more guilt about such aggrcssion, and longer duration of anger. No significant differences were found on general impulsiveness, either for women or for men.

The column in 'Table 3 which presents the significance of the sex-disease interaction shows that for most variables, the interaction is significant or near-significant. In general, where the women with RA tend to be more irritable and overtly aggressive than women without RA, the men with RA are less irritable and aggressive than the healthy men; this is especially true when the irritation or overt aggression involves the spouse. With regard to duration of anger, men with RA tend to remain angry a longer time, whereas women with RA get over their anger especially fast.

It may also be useful to contrast Table 3 with Table 2 . In both tables, the women with RA are more strongly differentiated from healthy women than are the arthritic men from the healthy ones. However, on the mental health variables, the differences between RA's and non-RA's are generally in the same direction for both sexes, whereas 


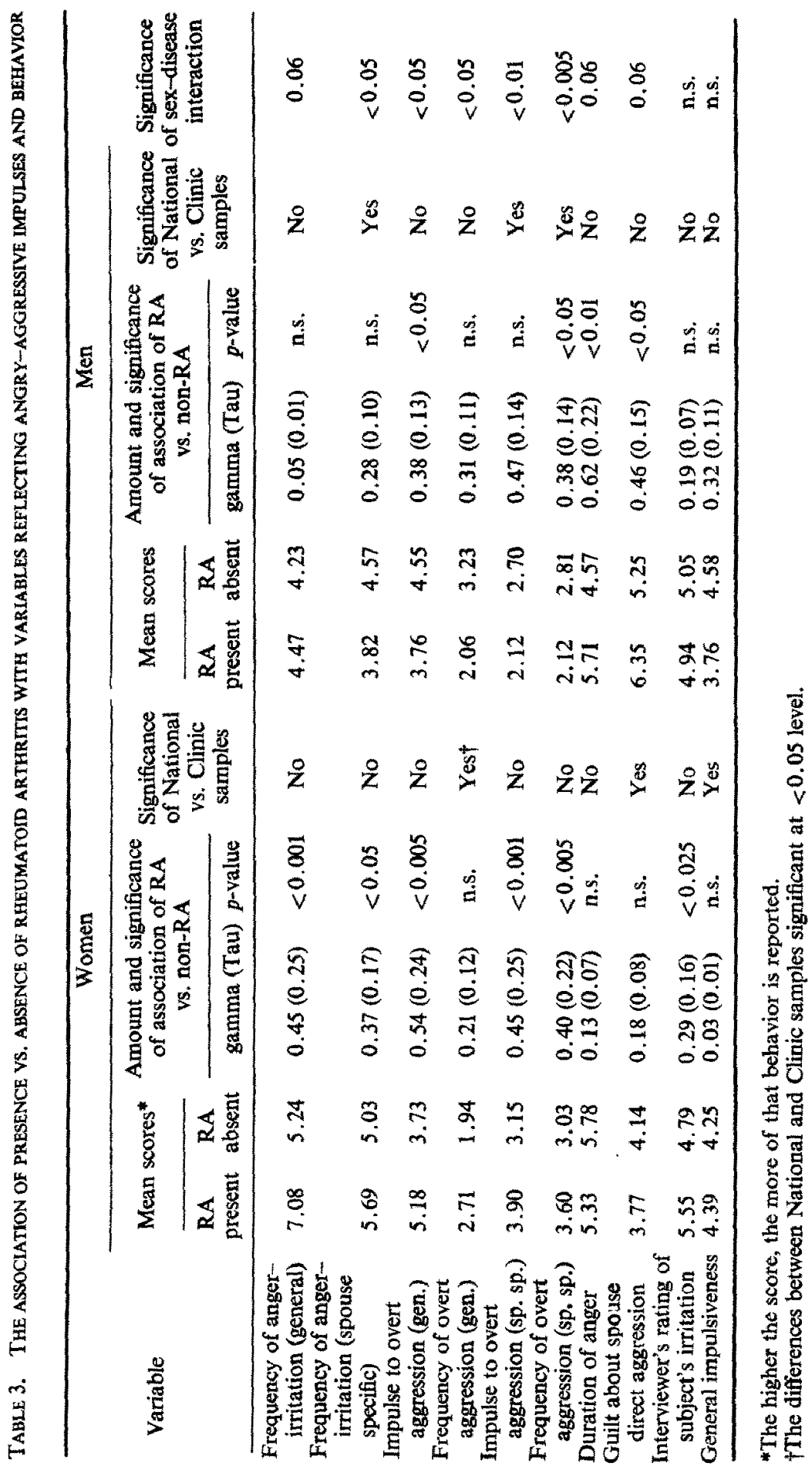




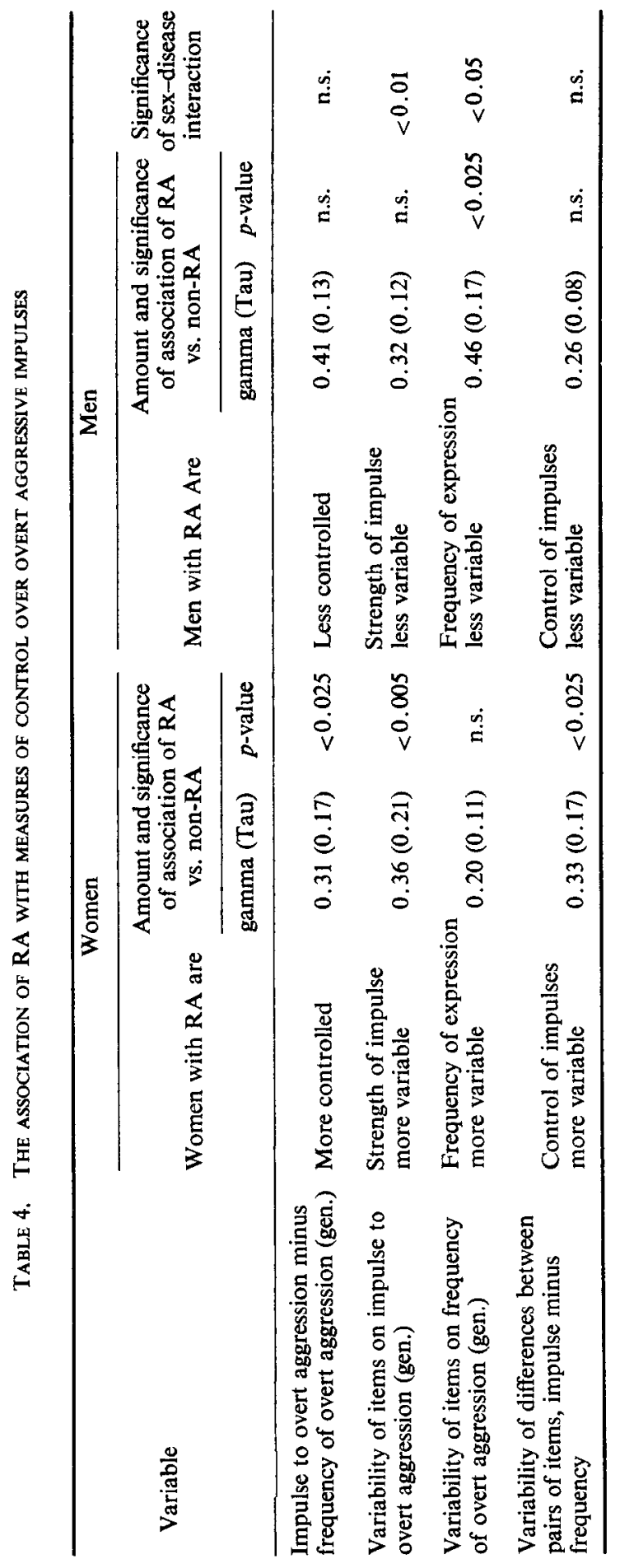


for the anger-aggression variables, the differences are, by and large, in opposite directions.

It will be seen in Table 3 that for a number of variables there are significant differences between National and Clinic samples. Adjustments for sample differences were made, as described above for Table 2, and, again, the new gamma and Tau values were substantially the same as the unadjusted ones. The significance of the sex-disease interaction was also basically unaltered.

One final comment on Tables 2 and 3. The measures of association for each of the variables in these two tables were recomputed after persons who might possibly have RA ( 22 women and 20 men) were taken out of the 'RA absent' category (where they are otherwise placed). The resultant gamma and Tau values for the significant associations were in most instances slightly higher and in the remainder, unchanged. Thus a more precise differentiation of the RA and the non-RA categories leads generally to a somewhat sharper separation along the psychological variables.

Let us now turn to the association of RA with some derived indices intended to describe more fully the conflict over expression of anger-aggression. First, we shall examine the difference scores obtained by subtracting 'frequency of overt aggression (general)' from 'impulse to overt aggression (general)'. The reader will recall that these scales contain identical items, except that one reflects the frequency of actual behavior, while the other indicates how often the respondent feels like doing something. The difference scores thus reflects the amount of control a person has over his aggressive impulses, the degree to which such impulses are blocked and never lead to overt aggression.

Table 4 presents the relevant data. The first variable in that table is the above mentioned difference score and it can be seen that women with RA are more controlled than women without RA (the gamma and Tau values become 0.36 and 0.19 , respectively, when adjustments are made for slight sample differences). Men with RA are less controlled than healthy men, but this difference is not significant, nor is the sex-disease interaction. The second and third variables in Table 4 were constructed to answer the question: Are there some subjects whose impulses to overt aggression, or frequency of actual aggression, is evenly distributed (i.e., is equally frequent) across different areas of behavior reflected in the different items, and is this systematically related to RA? From the table it can be seen that among women, such subjects are more likely to be non-RA's, while among men, they are more likely to bc RA's; the sex-disease interaction is significant for both variables. The last measure in Table 4 is trying to get at the 'evennesss' of the controls on aggressive impulses: high variability indicates that some kinds of aggressive impulses are particularly well controlled or blocked, while other impulses appear to escape control. Low variability, on the other hand, indicates that all kinds of aggressive impulses are controlled to the same degree. From the table it is apparent that women with RA have more uneven control over different kinds of aggressive impulses than do healthy women; among men, the association with RA is in the opposite direction but is not significant.

There is another pair of scales, 'impulse to overt aggression' and 'frequency of overt aggression' spouse specific), for which a difference score was also constructed. Such a score again reflects the amount of control a person has over his aggressive impulses, but now these impulses are directed at the spouse. Again, it was found that among women, the RA's were more controlled, while among men, the RA's were less 


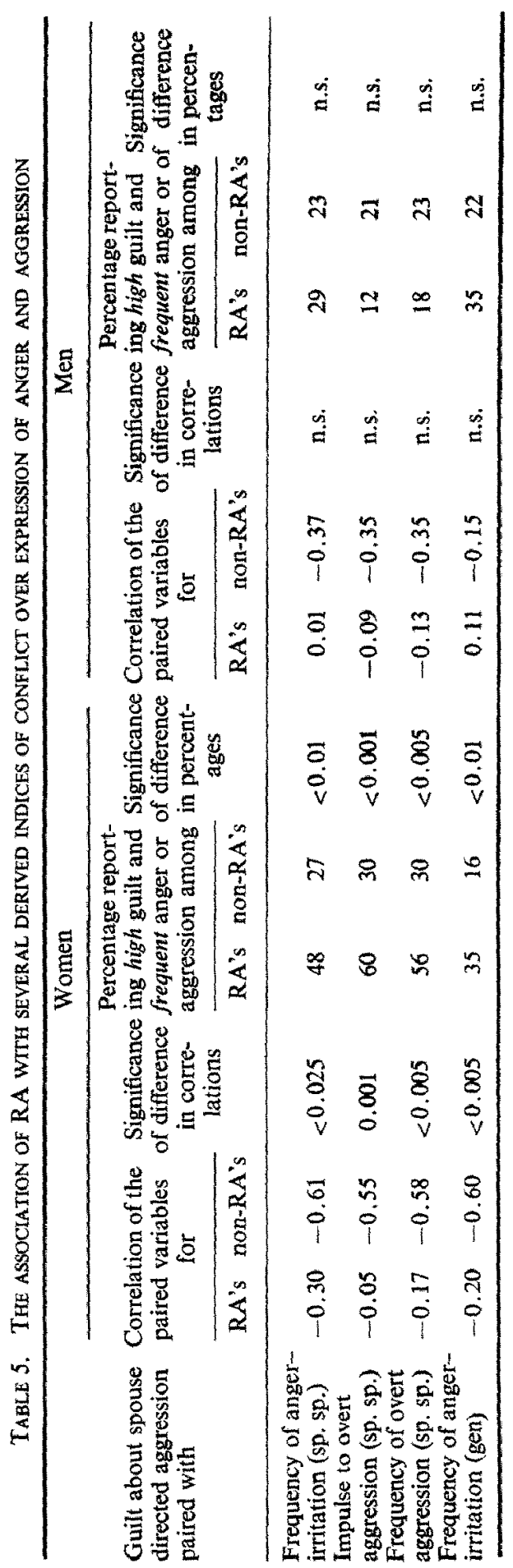


controlled. However, these differences were small and did not reach statistical significance.

Another set of derived indices consisting of pairing a focal variable, 'guilt about spouse directed aggression', with several other variables reflecting impulses to overt aggression or frequency of such aggression. The data are presented in Table 5. As can be seen, the measure of guilt is paired with 4 other variables, 3 of which are 'spouse specific' measures. The correlations between these 4 pairs of variables are given separately by sex and presence vs. absence of RA. Among women, all of the differences in the correlations are significant and in each case the non-RA's show a stronger negative relationship between guilt and anger or aggression or aggressive impulses. For the men, the differences between the RA's and non-RA's are in the same direction, but they are not significant. In the next step, the paired variables were approximately dichotomized and 2-by-2 tables were constructed. Among the women, it was found that the RA's were always unduly frequent in the quadrant representing high conflict, i.e., high guilt but relatively strong anger or aggression, or aggressive impulses. The percentages for the RA's and the non-RA's and the significance of the differences, are given in the table. Among the men, the RA's were not found unduly frequently in the same quadrant representing high conflict: the differences were small and inconsistent.

In the above pairing of variables, it was assumed that the combination of high aggression and strong values against it (high guilt) represents a conflict situation and it was found that the women with RA experience such a conflict more often. It is possible to pair other variables together and infer conflict, provided they also tend to imply incompatible behaviors. Thus, for example, among women with RA there is a sizeable correlation $(r=0.54)$ between the 'frequency of anger-irritation (general)' and the general 'need for emotional dependence'; among healthy women, the correlation is significantly smaller $(r=0.23)$. Now if is is assumed that one must curb one's irritability if others are to satisfy one's emotional dependence needs, then to be high on both variables represents a conflict situation. And of the women with RA, 42 per cent reported such implied conflict, whereas only 21 per cent of the healthy women were in the same situation, the difference being significant at the $<0.01$ level. Among the men, the RA's were not found significantly more frequently in such a conflict.

Another interesting comparison can be made between the 'frequency of angerirritation (general)' and the derived measure of control (used in Table 4), 'impulse to overt aggression' minus 'frequency of overt aggression'. In the original formulation of the frustration-aggression hypothesis [14] the authors suggest that inhibition of the aggressive impulse may be conceived as a frustration and, therefore, as an additional potential instigation to aggression. Thus we would expect a positive association between frequency of anger-irritation and strength of control (inhibition). The data mildly support this hypothesis; the correlations are 0.19 and 0.26 for women and men, respectively. The interesting finding is that the hypothesis is much better supported for the RA's; the correlations are $0.30(<0.025)$ and $0.77(<0.001)$ for female and male RA's respectively. Stated another way, among the female RA's there are more subjects for whom high control is accompanied by frequent angerirritation than among the healthy women (51 vs. 25 per cent, $p<0.001$ ). For men, the 


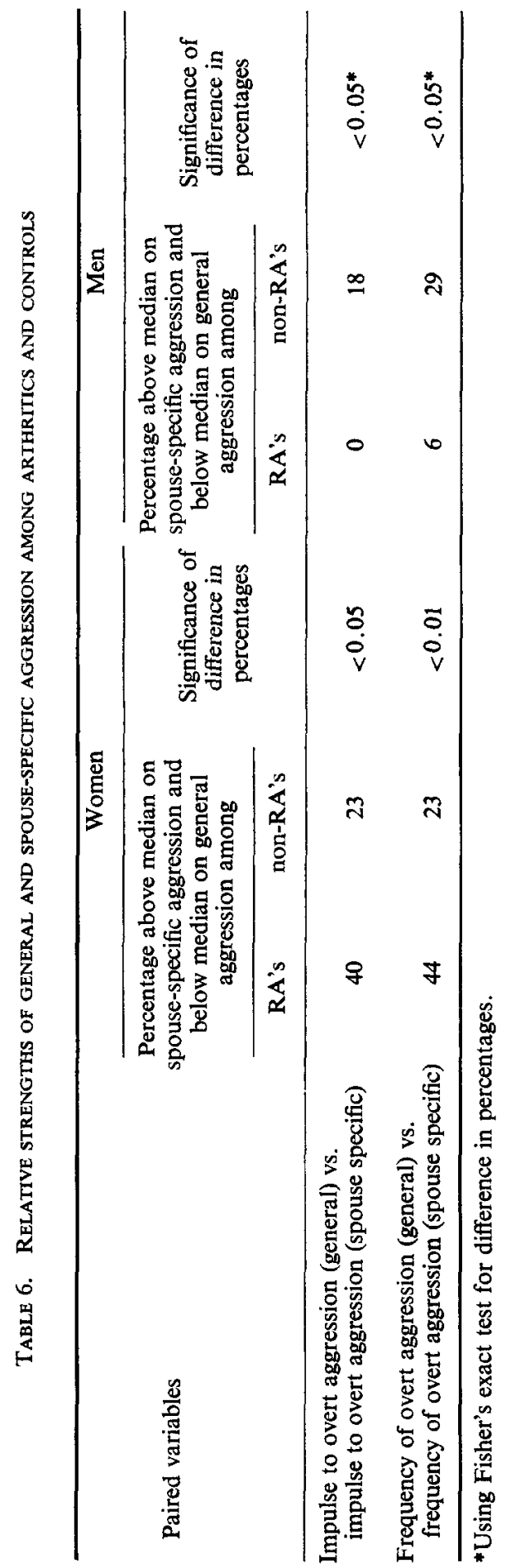


corresponding RA-non-RA percentages are 35 and 11 per cent $(p<0.01)$, respectively.

Our final examination of derived meaures involves the comparison of two pairs of scales: (a) 'impulse to overt aggression', general vs. spouse specific; $(b)$ 'frequency of overt aggression', general vs. spouse specific. The rationale for selecting these pairs of variables is the following: If we assume that one successful way of dealing with one's spouse-directed aggression is to displace it on other targets, then reporting more aggression on the general scale than on the spouse specific scale should be an indirect indication of a successful way of managing one's aggressive impulses. In other words, subjects whose spouse-specific aggression is relatively more frequent than their general (unspecified) aggression have apparently not found a successful solution and should be experiencing more conflict in the marriage.

Table 6 presents the relevant findings. It is apparent that among the women, the RA's are significantly more often than the non-RA's in that quadrant of a 2-by-2 table characterized by strong spouse-specific aggression but weak general aggression. Among the men, the difference between RA's and non-RA's is in the opposite direction and also significant.

So far, we have examined the association of a number of variables and of some derived indices with RA. In some of those measures, the subject reported on their general behavior, while in others, they were describing their behavior toward their spouse. To the extent that many of the spouse-specific measures yielded significant associations with RA, and some showed a sex-disease interaction, the inference may be drawn that the marriage involving an arthritic wife and a healthy husband may be different from the one where the husband has the RA, or where both are healthy. It is our purpose now to check more closely on this inference. The basic unit of analysis will be the marriage; we are dealing with 41 marriages where both partners are healthy, 28 marriages where the wife has RA and the husband is healthy, and 11 where the husband has RA and the wife is healthy. In this instance, 'healthy' means absence of RA and absence of ulcer. Since the seventh report in this series deals with the RA wife-ulcer husband relationship, the findings to be examined exclude the ulcer cases.

The major findings are given in Table 7. The first 4 columns give the correlations for the various variables and across the various types of marriages; the correlations reflect the amount of agreement between the husband's report on himself and the wife's report on herself. In general, the spouse-specific measures of anger or aggression tend to produce higher correlations than the general measures. Moreover, the correlations on the spouse-specific measures for the 'husband and wife healthy' marriage tend to be higher than the correlations for the "husband healthy and wife RA' marriage (the correlations for the third type of marriage are based on only 11 cases and vary a great deal). The last 2 variables in Table 7 are outside of the anger-suppression domain and are included merely to remind the reader that there are other variables which may show positive association across marriage. However, this is not the place for a thorough-going analysis of which mental health variables do or do not show such association.

The last 3 columns in Table 7 present the association of the various variables with the different types of marriages. The following procedure was used to compute the gamma and Tau values. The scores on each variable for the husband and the wife 


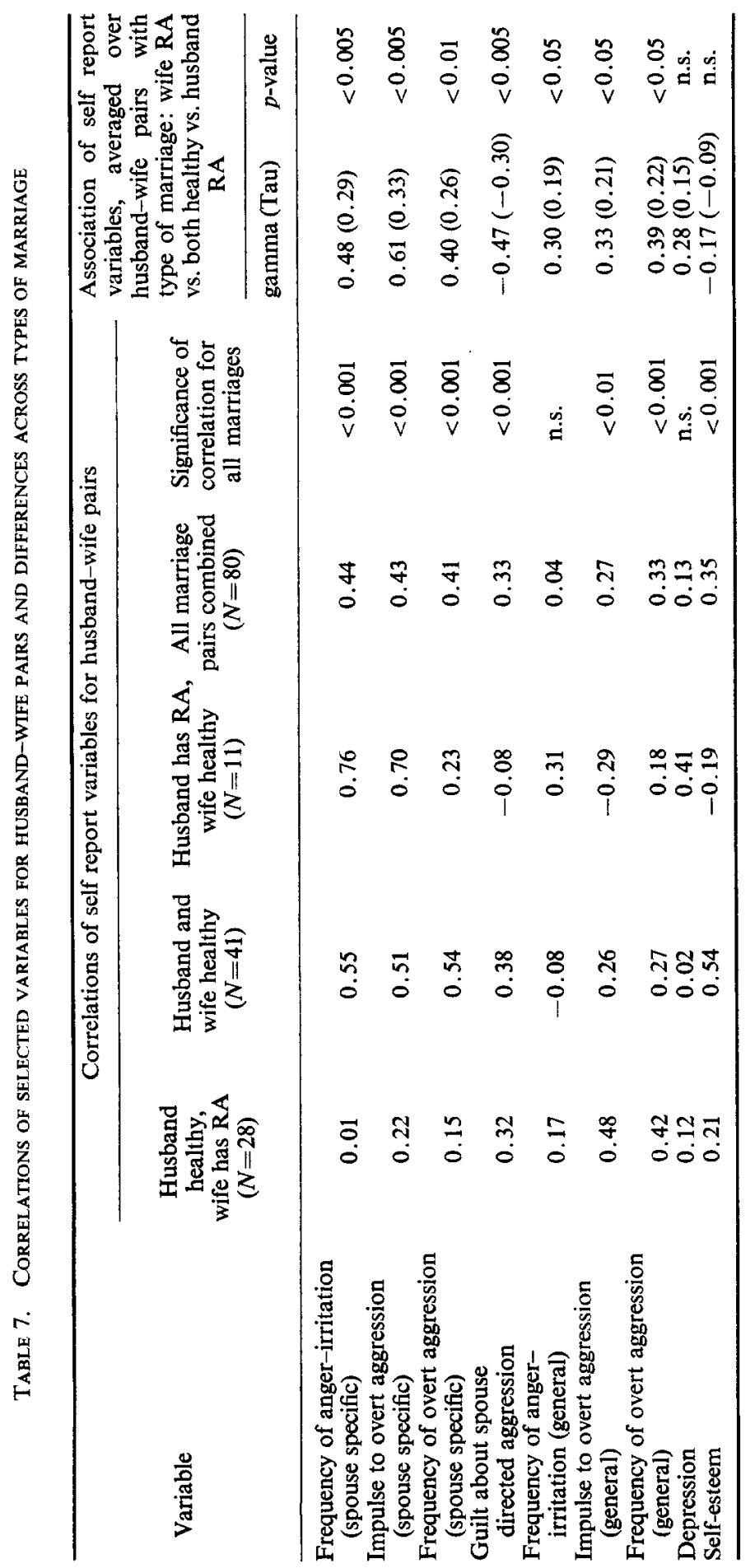


were averaged in order to get a single score, characterizing the marriage. Then the 3 types of marriage were ordered as follows: 'Wife RA and husband healthy' first, then 'husband and wife healthy', and then 'husband RA and wife healthy'. In this way, type of marriage can be treated as an ordered scale and high positive gamma (Tau) values indicate that the 'wife RA and husband healthy' marriages are the highest on the various measures, while the 'husband RA and wife healthy' marriages are the lowest. The obtained gamma (Tau) values in Table 7 are mostly positive and those dealing with anger-aggression are all significant. The one significant negative association for 'guilt about spouse directed aggression' fits in with the total pattern of findings: marriages higher on the various measures of aggression (i.e. 'wife RA and husband healthy') are lower on reported guilt.

\section{DISCUSSION}

In this section we shall be concerned with 3 issues: $(a)$ intercorrelations among the various variables; $(b)$ the possibility of contamination in our measures affecting the trustworthiness of the findings; and $(c)$ the interpretation of the results.

The major psychological variables, plus age, education, and income were all intercorrelated separately for men and women. The resulting 2 corrclation matrices were examined and can be summarized as follows:

(1) The correlations with the three demographic variables were generally negligible and not consistent across sex. Only 6 out of 114 correlations had an absolute value of 0.25 or higher, and the highest one was only 0.30 . Moreover, as the reader will recall, the design of this study minimizes differences between RA's and non-RA's on these demographic variables.

(2) There were three clusters of variables where moderately high within-cluster correlations and low across-cluster correlations could be seen: (a) One cluster included all of the scales dealing with anger-aggression; moreover, the general and the spousespecific scales tended to form 2 subclusters. (b) The second cluster included the several symptom scales ('physical ill health', 'physical anxiety', 'psychological anxiety', and 'immobilization') and the 'depression' scale. For the women, the within-cluster correlations were higher than for the men. $(c)$ The third cluster included 'self-esteem' (adult), 'self-confidence', and 'job satisfaction', and here the men showed higher within-cluster correlations.

The second issue under discussion is the possibility that therc arc extraneous factors contaminating our measures and therefore distorting the meaning of the findings. One such source of distortion might reside in the difference between the 2 sources of our subjects, National and Clinic samples. However, it was found that very few measures showed reliable differences between the 2 samples. Moreover, for those differences which were significant (and all were small), corrections were made before differences between RA's and non-RA's were examined. Finally, no measure showed a significant disease-sample interaction; that is, the RA vs. non-RA differences in the 2 samples were comparable and/or within sampling error.

Another source of contamination might have been due to high correlations with such demographic variables as age or social class. However, we have seen that such correlations were quite low and no general pattern was evident.

A still different potential problem is the possibility that individual differences in tendency to complain are a sufficient explanation of some of our findings. That is, 
if the RA measure reflects, in part, the tendency to complain, then any other measure, tapping the same tendency, would show some correlation with the RA measure. The second paper in this series takes up this issue at greater length and offers the tentative conclusion that the RA measure does not reflect the tendency to complain to any significant extent. Here we shall make 3 points in support of this conclusion: (1) Comparable differences between RA's and non-RA's were obtained in the National and Clinic samples. This would not be expected if only the Clinic cases were the true RA's while the National sample RA's were largely only complainers. (2) There was no overall significant association between RA and the 'physical ill health' variable (Table 2), nor was the association significant within the National or the Clinic samples. However, if complaining were involved, then this association, more than any other, should be a strong one. (3) Finally, if complaining were a contaminating factor we would expect to find a positive association, among the arthritics, between the severity of the disease and the various variables of the study. The relevant findings are presented in Table 8. Two indices of the severity of the disease are used, the 'activity index' and the 'disability index'. These measures, which were discussed in the second paper of this series, are moderately correlated, $r=0.47$. The correlations of these indices with the other variables are given for the 49 female arthritics; the correlations for the male arthritics are not given because such correlations, based on 17 cases, would be quite unstable estimates of the true amount of association. From Table 8 it can be seen that most of the correlations are quite low and that the highest ones relate in the opposite direction than predicted from the complaining hypothesis.

Table 8 merits a second glance for another reason. It might be argued that many of the variables we are studying, especially those having to do with anger-irritation, reflect affects or behaviors which are related to RA simply because they are a consequence of a frequently painful disease. This argument, however, is not supported

TABLE 8. THE association OF THE MAJOR VARIABles OF THE STUDY WITH THE ACTIVITY AND DISABILITY INDICES AMONG FEMALE ARTHRITICS

\begin{tabular}{lrr}
\hline & \multicolumn{2}{c}{ Correlation with } \\
\cline { 2 - 3 } Variable & Activity index & Disability index \\
\hline 'Physical ill health' & 0.15 & 0.01 \\
'Physical anxiety' & -0.23 & -0.22 \\
'Psychological anxiety' & -0.04 & 0.02 \\
'Immobilization' & 0.23 & 0.09 \\
Depression & 0.07 & -0.06 \\
Self-esteem (adult) & 0.01 & 0.15 \\
Self-confidence & 0.07 & 0.06 \\
Job satisfaction & 0.15 & 0.03 \\
Desire for change & 0.32 & 0.20 \\
Need for emotional dependence (general) & 0.07 & 0.10 \\
Frequency of anger-irritation (general) & -0.18 & $-\mathbf{0 . 3 6}$ \\
Frequency of anger-irritation (spouse specific) & -0.11 & $-\mathbf{0 . 2 6}$ \\
Impulse to overt aggression (general) & -0.07 & $-\mathbf{0 . 2 5}$ \\
Frequency of overt aggression (general) & 0.03 & -0.21 \\
Impulse to overt aggression (spouse specific) & -0.20 & $-\mathbf{0 . 3 3}$ \\
Frequency of overt aggression (spouse specific) & -0.12 & $-\mathbf{0 . 3 1}$ \\
Duration of anger & 0.25 & $\mathbf{0 . 0 3}$ \\
Guilt about spouse-directed aggression & -0.04 & $-\mathbf{0 . 0 5}$ \\
Impulsiveness & -0.02 & $-\mathbf{0 . 1 6}$ \\
\hline
\end{tabular}


by Table 8 where the severity of the disease is not accounting for the level of angerirritation or aggression in the predicted way. That is, women with more severe disease are reporting less frequent anger-irritation than those with mild disease. Thus anger-irritation discriminates best between healthy respondents and milder cases of RA.

The argument that anger-irritation or aggressive behavior are a consequence of the disease is also inadequate because it doesn't explain why the argument applies only to women. Male arthritics are lower on anger-aggression. Does it mean that for them the consequence of the disease is to lower anger-aggression? Finally, the argument is insufficient because it has been shown elsewhere [15] that the respondent's level of anger-aggression is related to status incongruence of parents. This is a variable which broadly describes the respondent's childhood environment and therefore is a variable which is located in time prior to the disease. Hence, part of angeraggression is anchored to a variable truly antecedent to the development of RA.

Another factor which could be contaminating our measures is respondents' defensiveness. That is, individual differences in defensiveness, or in the tendency to answer in the socially desirable way, could, if systematically related to RA, be underlying the observed association with RA. The problem of defensiveness is an everpresent difficulty in questionnaire measures but a recent examination of the issue $[16,17]$ suggests that the extent of the bias has been overestimated. Nevertheless, the problem is still there and even though we cannot estimate its extent in the present study, the following remarks seem appropriate.

(1) The fact that the husband's and the wife's self-reports show sizeable correlations with each other, especially in the healthy marriages (Table 7), suggests that more than defensiveness enters into the self-report measures. The assumption here is that through interaction and communication in the marriage, the husband and wife learn to reduce any large discrepancies between the amounts of aggression each expresses towards the other. It is, of course, still possible that they only learnt to become more similar to each other on defensiveness and then this similarity alone accounts for the observed husband-wife correlations on the self-report measures, but intuitively this seems less likely.

(2) Another relevant point is that in many of the analyses (e.g., Tables 4-6) we make use of derived measures, such as the difference score between 'impulse to- and frequency of overt aggression'. To the extent that we would expect defensiveness to operate equally on both scales, such a difference score becomes a kind of a selfanchoring scale with defensiveness being effectively ruled out.

(3) An examination of the pattern of findings would again suggest that something more than defensiveness is producing the results. For example, the scale called 'impulsiveness' correlates among the women, $r=0.47$, with 'impulse to overt aggression (general)'. The scales have a similar format and both deal with impulses which are not socially desirable. Yet on the former scale, the RA's show no differences from the non-RA's. while on the latter, the RA's are clearly higher. In fact, one can construct a difference score, the excess of specific aggressive impulses over general level of impulsiveness, and this self-anchoring scale discriminates the RA's from the non-RA's (gamma $=0.36(\mathrm{Tau}=0.20), p<0.01)$. An examination of another pair of scales, 'self-esteem (adult)' and job satisfaction, reveals a similar picture: two moderately correlated scales with content similar enough to suggest that compar- 
able amounts of defensiveness might be involved, yet only one discriminating women with RA from women without RA.

These arguments, of course, cannot alone dispose of the problem of defensiveness. Rather, they are intended to set some limits to the possible seriousness of the problem. As we shall see below in the interpretation of results, we cannot quite rule out the possibility that male RA's as a group are much more defensive about admitting to aggressive impulses.

Our last task is to comment about the overall meaning of our results. The major findings may be described as follows: (1) Both women and men with RA score higher on diverse measures of poor mental health than do respondents without RA. For the women, these differences are stronger and involve more diverse aspects of poor mental health. (2) Women with RA report more frequent anger-irritation and more frequent impulses to overt aggression; these differences hold irregardless of whether the anger-aggression is general or is directed at the husband. On measures of guilt about husband-directed aggression and duration of anger, women with RA were like the non-RA's. Men with RA, however, tended to differ from the healthy men in the opposite direction: they reported less wife-directed aggression, less frequent impulses to overt aggression, stronger guilt about aggression, and their anger episodes were of longer duration. (3) When a comparison was made of two parallel scales, frequency of impulses of overt aggression and frequency of actual aggressive acts, the women with RA: $(a)$ showed stronger inhibition or control of aggressive impulses (as reflected by a difference score between the two scales); but (b) seemed unable to apply the control to all types of aggressive impulses equally. The men with RA tended to be less controlled but were applying the controls particularly equally. However, since the differences among the men were not significant, the contrast here between men and women with RA is not as reliable. Moreover, in one respect, the RA men and women were alike in their difference from healthy respondents: for both of them, the inhibition of aggressive impulses fed back into their tendency to feel angry or irritated. (4) When the scale, 'guilt about spouse-directed aggression', was paired with measures of amount of spouse-directed anger or aggression, women with RA were unduly frequently found above median on both paired scales, i.e., reporting frequent husband-directed aggression and yet also relatively high guilt. It was suggested that this might indicate greater conflict among the RA women. No differences were found between men with and without RA. (5) When a comparison was made between 2 pairs of scales which were parallel in another way, impulses to aggression, general vs. spouse specific, and frequency of overt aggression, general vs. spouse-specific, then it was found that: (a) women with RA 'preferred' to direct their aggression at their husbands more often than women without RA; $(b)$ men with RA 'avoided' directing their aggression at their wives more often than men without RA. (6) Data analysis by husband-wife pairs showed that spouse-specific variables dealing with anger-aggression were positively correlated. As a consequence, healthy husbands of RA wivcs wcre higher on aggression than healthy husbands of healthy wives and healthy wives of RA men were lower on aggression than healthy wives of healthy husbands. It also follows that when scale scores were averaged for the spouses to yield a single marriage score, the wife RA-husband healthy marriages were the highest on anger-aggression, while the husband RA-wife healthy marriages were the lowest. 
The following general picture seems to be emerging. The RA women are more angry and irritable and experience stronger impulses to overt aggression. The target of this anger-aggression is the RA's husband as well as other people around her. In fact, the husband in a sense appears to be the 'preferred' target. But there are important exceptions to this general picture. The female RA's do not express their over aggression as often as the reported frequency of their impulses would demand. By implication, they exercise some control over the expression of these impulses and since the impulses are so frequent, their control is stronger than among the women without RA. This stronger control, however, is more erratic (less reliable) in the sense that some kinds of aggressive impulses escape control much better than other impulses. Another exception to the general picture is the fact that on 'guilt about spousedirected aggression', the female RA's are higher than they should be given their higher level of spouse-directed anger and aggression. Their guilt is not significantly different from the guilt of the non-RA's, but because they are more often angry at the husband or aggress more often against him, they presumably experience guilt more often. Thus, there is presumptive evidence that the higher anger and aggression of the female RA's is accompanied by some attempts at control and by a sense of guilt which apparently has not been blunted through frequent arousal.

The general picture of the male RA's is in some contrast to the description just given of the female RA's. Specifically, the male RA's, in comparison with the nonRA's, report less anger and less aggression, particularly when it is directed at the wife, and more guilt about such aggression. Thus, because the RA vs. non-RA differences are in the opposite directions for the 2 sexes, we get a significant sex-disease interaction for these variables.

However, this apparently simple contrast between male and female RA's encounters at least 2 problems. One problem stems from the fact that the male RA's report that when they get angry, their anger lasts a long time, i.e. their scores on 'duration of anger' are significantly higher than the scores for healthy men (Table 3). Yet we would expect the male RA's to report shorter-lasting anger because of this scale's positive correlations with other anger and aggression scales on which the RA's are low. For example, if we pair frequency of anger-irritation (general) with duration of anger, we find that 41 per cent of the RA's, but only 17 per cent of the non-RA's, report long-lasting but infrequent anger episodes; the difference is significant $(p<$ 0.025 ). Among the women, the RA's are more often found in the frequent-but-shortanger-episodes category than the non-RA's ( 35 vs. 13 per cent, $p<0.005$ ). Similarly, if we pair 'impulse to overt aggression (general)' with 'duration of anger,' we find that 53 per cent of the male RA's, but only 12 per cent of the non-RA's $(p<0.001)$, are in the long-lasting-anger-but infrequent-aggressive-impulses category. On the other hand, 46 per cent of the female RA's vs. 24 per cent of the non-RA's $(p<0.01)$, are in the frequent-aggressive-impulses-but-short-anger category. It would thus seem that if we think of the anger experience as frequency of angry episodes multiplied by their length, then the male and female RA's are 'quantitatively' similar but qualitatively different: their total time spent in anger episodes may be comparable, but for the women it is the frequency, while for the men it is the duration which contribute to the total time.

The other problem which complicates the apparently simple contrast between male and female RA's is the possibility that the picture of the male RA's as experiencing 
anger rarely and as being particularly low on aggressive impulses and overt aggression, is in some way misleading. It could be misleading in at least three ways: (1) Thcy are more unwilling to admit their anger-aggression to the interviewer than are other respondents. (2) They lack awareness of their own angry feelings and their aggressive impulses, but these are present and do get expressed. (3) They inhibit anger and aggressive impulses so strongly that they neither express nor report them. (See Nemiah's case of Gordon C. [18]).

Little evidence can be cited to help the reader decide whether to accept the apparent meaning of the data describing the male RA's or whether to accept 1 of the above 3 skeptical alternatives. However, it does seem to us that the data on the wives of the male RA's - the wives are low on aggression and their own level of anger-aggression correlates well with the husband's (Table 7)-favor the last of the 3 alternatives more than the other 2. The reader will also appreciate the fact that in trying to choose between 2 alternative explanations - the male RA's experience anger and aggressive impulses rarely vs. the male RA's are so inhibited that they neither feel their anger nor do they overtly express their aggressive impulses-one is demanding of one's assessment procedures much more sophistication and subtlety than is easily obtained in interview scales.

\section{SUMMARY}

This study describes the differences between adult rheumatoid arthritics (RA's) and controls on a number of personality variables. The cases come both from a national interview sample and a university arthritis clinic, while the controls were part of a cluster which included the spouse of the RA, the RA's sib and his (her) spouse, a cousin of the RA, and an unrelated individual. The data were collected in the course of three structured interviews. The major findings werc the following:

(1) RA's of both sexes score higher on several measures of poor mental health than the non-RA's. For the women, these differences are stronger and involve more diverse aspects of poor mental health.

(2) Women with RA report more frequent anger-irritation and more frequent impulses to overt aggression than women without RA. Involved in these differences are scales reflecting both general anger-aggression and that which is directed at the husband.

(3) Men with RA, compared with healthy men, report less frequent impulses to general overt aggression, less wife-directed aggression, stronger guilt about such aggression, and their anger episodes were of longer duration.

(4) When a difference score was computed between two scales, frequency of impulses to overt aggression and frequency of actual aggressive acts, women with RA, compared with healthy women, showed stronger control over the expression of such impulses. but seemed unable to apply the control to all types of aggressive impulses equally.

(5) Women with $\mathrm{R \Lambda}$ were unduly frequently found above median on 'guilt about spouse-directed aggression' and above median on several measures of husbanddirected anger-aggression. It was suggested that this might indicate greater conflict among the RA women.

(6) Comparing general and spouse-specific measures of overt aggression, (a) women with RA 'preferred' to direct their aggression at their husbands more than 
healthy women; while $(b)$ men with RA 'avoided' directing their aggression at their wives more often than healthy men.

(7) When husbands' self-reports of wife-directed anger-aggression were correlated with wives' self-reports of husband-directed anger-aggression, the associations were positive and moderate to strong.

(8) Marriages of RA wives and healthy husbands were highest on mutually directed anger-aggression; marriages of RA husbands and healthy wives were the lowest, while the healthy husbands-healthy wives marriages were intermediate.

\section{REFERENCES}

1. KING, S. H.: Psychosocial factors associated with rheumatoid arthritis: An evaluation of the literature. J. chron. Dis. 2, 287, 1955.

2. Moos, R. H.: Personality factors associated with rheumatoid arthritis; A review. J. chron. Dis. $17,41,1964$.

3. SсотсH, N. A. and Geiger, H. J.: The epidemiology of rheumatoid arthritis: $\Lambda$ review with special attention to social factors. J. chron. Dis. 15, 1037, 1962.

4. CAmpreLl, D. T. and Fiske, D. W.: Convergent and discriminant validation by the multitraitmultimethod matrix. Psychol. Bull. 56, 81, 1959.

5. CoBb, S. and KASL, S. V.: Epidemiologic contributions to the etiology of rheumatoid arthritis with special attention to psychological and social factors. Chap 12 in: Population Studies of the Rheumatic Diseases (Ed. by P. H. BeNNETT and P. H. N. WoOD) Excerpta Medica, Amsterdam, 1968.

6. Gurin, G., Veroff, J. and Feld, S.: Americans View their Mental Health. Basic Books, New York, 1960.

7. French, J. R. P., JR. and KAHN, R. L.: A programmatic approach to studying the environment and mental health. J. Soc. Issues 18, (3) 1, 1962.

8. Scotr, W. A.: Research definitions of mental health and mental illness. Psychol. Bull. 55, 29, 1958.

9. Scotr, W. A.: Social psychological correlates of mental illness and mental health. Psychol. Bull. $55,65,1958$.

10. Cobb, S., Kasl, S. V., Chen, E. and Christenfeld, R.: Some psychological and social characteristics of patients hospitalized for rheumatoid arthritis, hypertension and duodenal ulcer. J. chron. Dis. 18, 1259, 1965.

11. SAmpson, E. E.: An Experiment on Active and Passive Resistance to Social Power. Unpublished doctoral dissertation, The University of Michigan, 1960.

12. KasL, S. V., SAmpson, E. E. and French, J. R. P., JR.: The development of a projective measure of the need for independence: a theoretical statement and some preliminary evidence. $J$. Personality 32, 566, 1964.

13. Scort, W. A. and WeRThermer, M.: Introduction to Psychological Research. Wiley, New York, 1962.

14. Dollard, J., Doob, L. W., Miller, N. E., Mowrer, O. H. and Sears, R. R.: Frustration and Aggression. Yale University Press, New Haven, 1939.

15. KASL, S. V. and Совв, S.: The effects of parental status incongruence and discrepancy on physical and mental health of adult offspring. J. Personality soc. Psychol. Mongr. 7, 1, No. 2 (Whole No. 642) 1967.

16. Block, J.: The Challenge of Response Sets. Appleton-Century-Crofts, New York, 1965.

17. Norman, W. T.: On estimating psychological relationships: Social desirability and self-report. Psychol. Bull. 67, 273, 1967.

18. Nemia, J. C.: Foundations of Psychopathology. Oxford University Press, New York, 1961. 